ASC Report No. 11/2007

Numerical Simulation of Thermal Effects in Coupled Optoelectronic Device-circuit Systems

Markus Brunk, Ansgar Jüngel 


\section{Most recent ASC Reports}

10/2007 Ansgar Jüngel, Ingrid Violet

First-Order Entropies for the Derrida-Lebowitz-Speer-Spohn Equation

09/2007 Maike Schulte, Anton Arnold

Discrete Transparent Boundary Conditions for the Schrödinger Equation - a Compact Higher Order Scheme

08/2007 Anton Arnold, Jose Antonio Carrillo, Corinna Klapproth Improved Entropy Decay Estimates for the Heat Equation

07/2007 Christoph Erath, Samuel Ferraz-Leite, Stefan Funken, Dirk Praetorius Energy Norm Based A Posteriori Error Estimation for Boundary Element Methods in Two Dimensions

06/2007 Anton Arnold, Irene M. Gamba, Maria Pia Gualdani, Christof Sparber The Wigner-Fokker-Planck Equation: Stationary States and Large Time Behavior

05/2007 Anton Arnold, Maike Schulte

Transparent Boundary Conditions for Quantum-Waveguide Simulations

04/2007 Anton Arnold, Jean-Philippe Bartier, Jean Dolbeault

Interpolation between Logarithmic Sobolev and Poincaré Inequalities

03/2007 Tareq Amro, Chunxiong Zheng

A PML Absorbing Boundary Condition for the Nonlinear Euler Equations in Unbounded Domains

02/2007 Christoph Erath, Dirk Praetorius

A Posteriori Error Estimate and Adaptive Mesh-Refinement for the Cell-Centered Finite Volume Method for Elliptic Boundary Value Problems

01/2007 Samuel Ferraz-Leite, Dirk Praetorius

Simple A Posteriori Error Estimators for the h-Version of the Boundary Element Method

Institute for Analysis and Scientific Computing

Vienna University of Technology

Wiedner Hauptstraße 8-10

1040 Wien, Austria

E-Mail: admin@asc.tuwien.ac.at

WWW: http://www.asc.tuwien.ac.at

FAX: $\quad+43-1-58801-10196$

ISBN 978-3-902627-00-1

(C) Alle Rechte vorbehalten. Nachdruck nur mit Genehmigung des Autors.

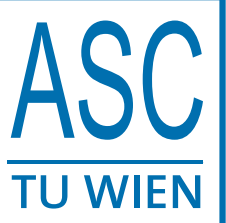




\title{
Numerical Simulation of Thermal Effects in Coupled Optoelectronic Device-circuit Systems
}

\author{
Markus Brunk $^{1}$ and Ansgar Jüngel ${ }^{2}$ \\ 1 Institut für Mathematik, Universität Mainz, Staudingerweg 9, 55099 Mainz, \\ Germany, e-mail: brunk@mathematik.uni-mainz.de \\ ${ }^{2}$ Institut für Analysis und Scientific Computing, Technische Universität Wien, \\ Wiedner Hauptstr. 8-10, 1040 Wien, Austria, \\ e-mail: juengel@anum.tuwien.ac.at
}

\section{Introduction}

The control of thermal effects becomes more and more important in modern semiconductor circuits like in the simplified CMOS transceiver representation described by U. Feldmann in the above article Numerical simulation of multiscale models for radio frequency circuits in the time domain. The standard approach for modeling integrated circuits is to replace the semiconductor devices by equivalent circuits consisting of basic elements and resulting in so-called compact models. Parasitic thermal effects, however, require a very large number of basic elements and a careful adjustment of the resulting large number of parameters in order to achieve the needed accuracy.

Therefore, it is preferable to model those semiconductor devices which are critical for the parasitic effects by semiconductor transport equations. The transport of electrons in the devices is modeled here by the one-dimensional energy-transport model allowing for the simulation of the electron temperature. The electric circuits are described by modified nodal analysis. Thus, the devices are modeled by (nonlinear) partial differential equations, whereas the circuit is described by differential-algebraic equations. The coupled model, which becomes a system of (nonlinear) partial differential-algebraic equations, is numerically discretized in time by the 2-stage backward difference formula (BDF2), since this scheme allows to maintain the M-matrix property, and the semi-discrete equations are approximated by a mixed finite-element method.

The objective is the simulation of a benchmark high-frequency transceiver circuit, using a laser diode as transmitter and a photo diode as receiver. The optical field in the laser diode is modeled by recombination terms and a rate equation for the number of photons in the device. The optical effects in the photo diode are described by generation terms. The numerical results show that the thermal effects can modify significantly the behavior of the transmitter circuit. 


\section{Modeling}

Circuit modeling. A well-established mathematical description of electric circuits, consisting of resistors, capacitors, and inductors (RCL circuit) is the modified nodal analysis (MNA) which can be easily extended to circuits containing semiconductor devices. In the following, the circuit model is described.

The circuit is replaced by a directed graph. The RLC branches are characterized by the incidence matrix $A$, and the semiconductor branches are characterized by the semiconductor incidence matrix $A_{S}$. The basic tools for the MNA are the Kirchhoff laws and the current-voltage characteristics for the basic elements,

$$
A i+A_{S} j_{S}=0, \quad v=A^{\top} e, \quad i_{R}=g\left(v_{R}\right), \quad i_{C}=\frac{d q}{d t}\left(v_{C}\right), \quad v_{L}=\frac{d \Phi}{d t}\left(i_{L}\right),
$$

where $i, v$, and $e$ are the vectors of branch currents, branch voltages, and node potentials, respectively, and $j_{S}$ denotes the semiconductor current (see below). The variable $g$ denotes the conductivity of the resistor, $q$ is the charge of the capacitor, and $\Phi$ the flux of the inductor. The incidence matrix $A$ is assumed to consist of the block matrices $A_{R}, A_{C}, A_{L}, A_{i}$, and $A_{v}$, where the indices $i$ and $v$ indicate the current source and voltage source branches, respectively.

Denoting by $i_{s}=i_{s}(t)$ and $v_{s}=v_{s}(t)$ the given input functions for the sources, we obtain the system for the charge-oriented MNA [13],

$$
\begin{aligned}
& A_{C} \frac{d q}{d t}\left(A_{C}^{\top} e\right)+A_{R} g\left(A_{R}^{\top} e\right)+A_{L} i_{L}+A_{v} i_{v}+A_{S} j_{S}=-A_{i} i_{s}, \\
& \frac{d \Phi}{d t}\left(i_{L}\right)-A_{L}^{\top} e=0, \quad A_{v}^{\top} e=v_{s},
\end{aligned}
$$

for the unknowns $e(t), i_{L}(t)$, and $i_{v}(t)$. Equation (1) expresses the Kirchhoff current law, the first equation in (2) is the voltage-current characteristic for inductors, and the last equation allows to compute the node potentials.

Semiconductor device modeling. The flow of minority charge carriers (holes) in the device is modeled by the drift-diffusion model for the hole density $p$. The electron flow is described by the energy-transport equations [8]. The first model consists of the conservation law for the hole mass, together with a constitutive relation for the hole current density. The latter model also includes the conservation law for the electron energy and a constitutive relation for the energy flux. Both models can be derived from the semiconductor Boltzmann equation (see [8] and references therein). They are coupled through recombination-generation terms and the Poisson equation for the electric potential. More precisely, the electron density $n$, the hole density $p$, and the electron temperature $T$ are obtained from the parabolic equations

$$
\begin{aligned}
& \mu_{n}^{-1} \partial_{t} g_{1}-\operatorname{div} J_{n}=-R\left(\mu_{n}^{-1} g_{1}, p\right), \quad \partial_{t} p+\operatorname{div} J_{p}=-R\left(\mu_{n}^{-1} g_{1}, p\right) \\
& \mu_{n}^{-1} \partial_{t} g_{2}-\operatorname{div} J_{w}=-J_{n} \cdot \nabla V+W\left(\mu_{n}^{-1} g_{1}, T\right)-\frac{3}{2} T R\left(\mu_{n}^{-1} g_{1}, p\right),
\end{aligned}
$$


where $g_{1}=\mu_{n} n$ and $g_{2}=\mu_{n} w$ are auxiliary variables allowing for a driftdiffusion-type formulation of the fluxes [8], $w=\frac{3}{2} n T$ is the thermal energy, and $\mu_{n}$ and $\mu_{p}$ are the electron and hole mobilities, respectively. The electron current density $J_{n}$, the energy flux $J_{w}$, and the hole current density $J_{p}$ are given by

$$
J_{n}=\nabla g_{1}-\frac{g_{1}}{T} \nabla V, \quad J_{w}=\nabla g_{2}-\frac{g_{2}}{T} \nabla V, \quad J_{p}=-\mu_{p}(\nabla p+p \nabla V) .
$$

The equations are coupled self-consistently to the Poisson equation for the electric potential $V$,

$$
\lambda^{2} \Delta V=\mu_{n}^{-1} g_{1}-p-C(x),
$$

where $\lambda$ is the scaled Debye length and the given function $C(x)$ models the doping profile. The functions

$$
W(n, T)=-\frac{3}{2} \frac{n\left(T-T_{L}\right)}{\tau_{0}} \quad \text { and } \quad R(n, p)=\frac{n p-n_{i}^{2}}{\tau_{p}\left(n+n_{i}\right)+\tau_{n}\left(p+n_{i}\right)}
$$

with the (scaled) energy relaxation time $\tau_{0}$ and lattice temperature $T_{L}=$ 1 describe the relaxation to the equilibrium energy and Shockley-Read-Hall recombination-generation processes with intrinsic density $n_{i}$ and electron and hole lifetimes $\tau_{n}$ and $\tau_{p}$, respectively.

Equations (3)-(6) are solved in the bounded semiconductor domain $\Omega$, where some initial values $n_{I}, p_{I}$, and $T_{I}$ are imposed. The boundary of $\Omega$ is assumed to split into two parts. On the insulating parts of the boundary $\Gamma_{N}$, it is assumed that the normal components of the current densities and of the electric field vanish. For the temperature, homogenous Neumann boundary conditions are assumed as in [1]. We have shown in [5] that boundary layers for the particle densities can be avoided if Robin-type boundary conditions similar as in [14] are employed on the remaining boundary parts,

$$
n-\theta_{n} J_{n} \cdot \nu=n_{a} \quad \text { and } \quad p+\theta_{p} J_{p} \cdot \nu=p_{a} \quad \text { on } \partial \Omega \backslash \Gamma_{N},
$$

where $\theta_{n}$ and $\theta_{p}$ are some parameters and $n_{a}$ and $p_{a}$ are ambient particle densities. Notice that in the one-dimensional simulations presented below, $\Gamma_{N}=\emptyset$.

Coupling to the circuit. The boundary conditions for the electric potential at the contacts are determined by the circuit and are given as

$$
V=e_{i}+V_{\mathrm{bi}} \quad \text { on } \Gamma_{k}, t>0, \quad \text { where } \quad V_{\mathrm{bi}}=\operatorname{arsinh}\left(\frac{C}{2 n_{i}}\right),
$$

if the terminal $k$ of the semiconductor is connected to the circuit node $i$.

The semiconductor current entering the circuit consists of the electron current $J_{n}$, the hole current $J_{p}$, and the displacement current $J_{d}=-\lambda^{2} \partial_{t} \nabla V$, guaranteeing charge conservation. The current leaving the semiconductor device at terminal $k$, corresponding to the boundary part $\Gamma_{k}$, is defined by 


$$
j_{k}=\int_{\Gamma_{k}}\left(J_{n}+J_{p}+J_{d}\right) \cdot \nu d s,
$$

where $\nu$ is the exterior unit normal vector to $\partial \Omega$. We denote by $j_{S}$ the vector of all terminal currents except the reference terminal. In the one-dimensional case, there remains only one terminal, and the current through the terminal at $x=0$ is given by

$$
j_{S}(t)-\left(J_{n}(0, t)+J_{p}(0, t)-\partial_{t} j_{d, S}(0, t)\right)=0, \quad j_{d, S}-\lambda^{2} V_{x}=0,
$$

where the circuit equations (1)-(2) have to be appropriately scaled [6].

The complete coupled system consists of equations (1)-(10) forming an initial boundary-value problem of partial differential-algebraic equations. The system resulting from the coupled circuit drift-diffusion equations has at most index 2 and it has index 1 under some topological assumptions [3, 13]. No analytical results are available for the coupled circuit energy-transport system.

Optoelectronic device modeling. The interaction between optical and electrical effects is modeled by recombination-generation terms appearing in (7). In the following, we present the model used in the numerical simulations and we refer to [6] for a discussion about the model simplifications.

For a vertical photo diode, the supplied photons generate free charge carriers generating the photo current. We model this effect by adding to the Shockley-Read-Hall term (7) the generation rate $G_{\text {opt }}(x)$ of free carriers at depth $x$, caused by the (scaled) optical irradiation power $P_{\text {in }}$ with angular frequency $\omega[10]$,

$$
G_{\mathrm{opt}}(x)=\eta(1-r) \frac{P_{\mathrm{in}}}{\hbar \omega A} \alpha_{\mathrm{ab}} e^{-\alpha_{\mathrm{ab}} x},
$$

where the physical parameters are the quantum efficiency $\eta$, the reflectivity $r$ of the irradiated surface with area $A$, the reduced Planck constant $\hbar=h / 2 \pi$, and the optical absorption $\alpha_{\mathrm{ab}}$.

The laser diode is modeled by a pin heterostructure diode in which the intrinsic (active) region consists of a low-band gap material causing carrier confinement. The active region works as a Fabry-Perot laser cavity and can be modeled as a single mode laser. The band discontinuities are simply described by adding a constant band potential to the electric potential $V$ in the active region [9]. Additionally to (7), spontaneous and stimulated recombination is introduced,

$$
R_{\mathrm{spon}}=B n p \quad \text { and } \quad R_{\mathrm{stim}}=\frac{c}{\mu_{\mathrm{opt}}} g(n)|\Xi|^{2} S,
$$

respectively, where $B$ is the spontaneous recombination parameter, $c$ the speed of light $\mu_{\mathrm{opt}}$ the refractive index of the material, $g(n)$ the optical gain depending on the electron density, $|\Xi|^{2}$ the intensity distribution of the optical field, which is a solution of the waveguide equation [6], and $S=S(x, t)$ is the number of photons in the device. 
The optical gain is approximated by $g(n)=g_{0}\left(n-n_{\mathrm{th}}\right)[7]$, with differential gain $g_{0}$ and threshold density $n_{\mathrm{th}}$. In the lasing mode we can employ the quasineutral assumption $n \approx p$ in the active region such that the gain becomes approximately $g(p)=g_{0}\left(p-n_{\mathrm{th}}\right)$ in the recombination term occurring in the hole equation (3). This allows for a discretization that guarantees positivity of the discretized hole density [6]. The number of photons $S$ is balanced by the rate equation

$$
\partial_{t} S=v_{g}(\beta-\alpha) S+R_{\mathrm{spon}}, \quad \text { where } \quad \beta=\int_{\Omega_{a}}\left(g(n)-\alpha_{\mathrm{bg}}\right)|\Xi|^{2} d s,
$$

$\alpha$ is the total loss by external output and scattering, $\alpha_{\mathrm{bg}}$ denotes the background loss, and $\Omega_{a}$ is the transverse cross section of the active region. We prescribe the initial condition $S(\cdot, 0)=S_{I}$ in $\Omega$. Finally, the output power is computed from of the number of photons by

$$
P_{\mathrm{out}}=\hbar \omega \frac{c}{\mu_{\mathrm{opt}}} \alpha_{f}|\Xi|^{2} S
$$

(see [2]), where $\alpha_{f}$ denotes the facet loss of the laser cavity.

\section{Numerical Simulations}

The system of coupled partial differential-algebraic equations is first discretized in time by the BDF2 method since this scheme allows to maintain the M-matrix property of the final discrete system. The Poisson equation is discretized in space by the linear finite-element method. Then the discrete electric potential is piecewise linear and the approximation of the electric field $-V_{x}$ is piecewise constant.

The semi-discrete continuity equations at one time step are of the form

$$
-J_{j, x}+\sigma_{j} g_{j}=f_{j}, \quad J_{j}=g_{j, x}-\frac{g_{j}}{T} V_{x}, \quad j=1,2,
$$

with the current densities $J_{1}=J_{n}$ and $J_{2}=J_{w}$ and some expressions $\sigma_{j}$ and $f_{j}$. These equations are discretized in space by a hybridized exponentially fitted mixed finite-element method [8]. We employ the finite elements of [12] since they guarantee the positivity of the discrete variables if positive initial and Dirichlet boundary data are prescribed and if $\sigma_{j} \geq 0, f_{j} \geq 0$ for $j=1,2$. This property also holds for the Robin conditions (8) [6]. Finally, the nonlinear discrete system is solved by Newton's method.

Rectifying circuit. As a test example we consider a rectifying circuit containing four silicon $p n$ diodes as in [5] (Fig. 1). Each of the diodes has the length $L=0.1 \mu \mathrm{m}$ (and $L_{y}=0.1 \mu \mathrm{m}, L_{z}=2 \mu \mathrm{m}$ ) or $L=1 \mu \mathrm{m}$ (and $\left.L_{y}=1 \mu \mathrm{m}, L_{z}=20 \mu \mathrm{m}\right)$ and a maximum doping of $10^{22} \mathrm{~m}^{-3}$. We have chosen the resistance $R=100 \Omega$ and the voltage source $v(t)=U_{0} \sin (2 \pi \omega t)$ 

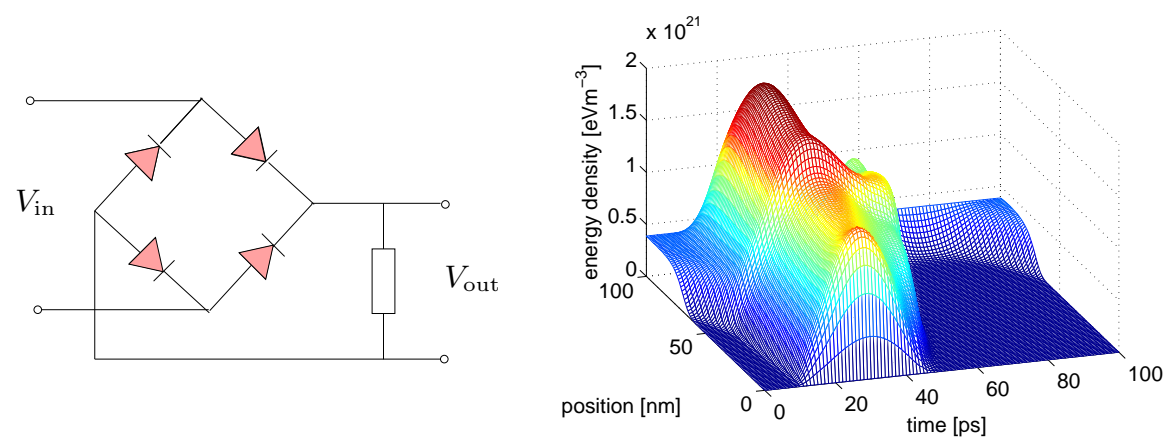

Fig. 1. Left: Graetz circuit. Right: Thermal energy in a $p n$ diode during one oscillation of $V_{\text {in }}$.

\begin{tabular}{|l|l|l|}
\hline Parameter & Physical meaning & Numerical value \\
\hline$q$ & elementary charge & $1.6 \cdot 10^{-19} \mathrm{As}$ \\
$\epsilon_{s}$ & permittivity constant & $1.05 \cdot 10^{-10} \mathrm{As} / \mathrm{Vm}$ \\
$U_{T}$ & thermal voltage at $300 \mathrm{~K}$ & $0.026 \mathrm{~V}$ \\
$\mu_{n} / \mu_{p}$ & low-field carrier mobilities & $1500 / 450 \mathrm{~cm}^{2} / \mathrm{Vs}$ \\
$\tau_{n} / \tau_{p}$ & carrier lifetimes & $10^{-6} / 10^{-5} \mathrm{~s}$ \\
$n_{i}$ & intrinsic density & $10^{16} \mathrm{~m}^{-3}$ \\
$\tau_{0}$ & energy relaxation time & $4 \cdot 10^{-13} \mathrm{~s}$ \\
\hline
\end{tabular}

Table 1. Physical parameters for a silicon $p n$-junction diode.

with $U_{0}=5 \mathrm{~V}$ and $\omega=1 \mathrm{GHz}$ or $\omega=10 \mathrm{GHz}$. The remaining physical parameters are listed in Table 1. As initial conditions we take thermal equilibrium densities in the device and vanishing node potentials and branch currents in the circuit. The initial value for the displacement current is determined by (10). A computation according to [11] shows that these values are consistent for the coupled DAE system.

In Fig. 1 the energy density in one of the diodes during one oscillation is presented. As expected, we observe a high thermal energy in forward bias $(t \in[0,50 \mathrm{ps}])$, whereas it is negligible in backward bias $(t \in[50 \mathrm{ps}, 100 \mathrm{ps}])$ although the electron temperature (not shown) may be very large around the junction [4].

The impact of the thermal effects on the electrical behavior of the circuit is shown in Fig. 2. The figure clearly shows the rectifying behaviour of the cuircuit. The largest current is obtained from the drift-diffusion model since we have assumed a constant electron mobility such that the drift is unbounded with respect to the modulus of the electric field. The stationary energy-transport model is not able to catch the capacitive effect at the junction which is particularly remarkable at higher frequencies.

Optoelectronic circuit. Next we consider a AlGaAs/GaAs laser diode with a digital input signal. The transmitted signal is received by a silicon photo 

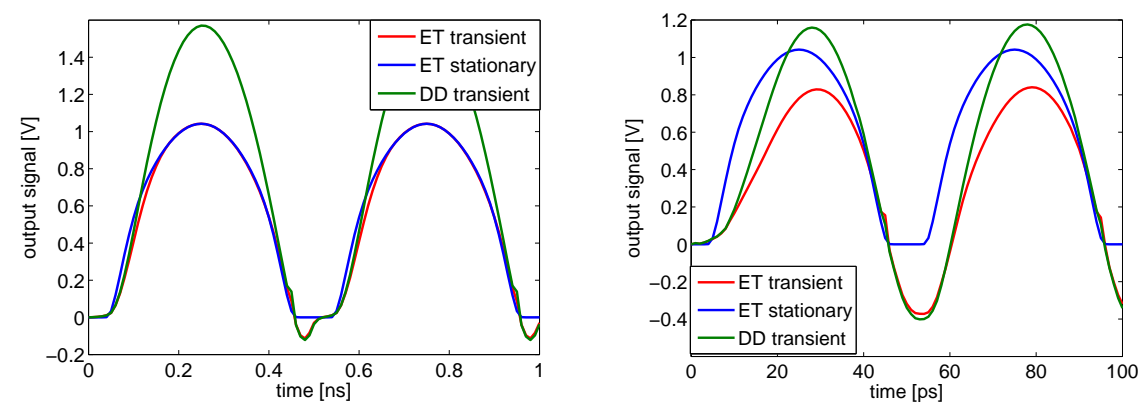

Fig. 2. Output signal of the Graetz circuit for two frequencies of the voltage source. Left: $\omega=1 \mathrm{GHz}$ and $L=0.1 \mu \mathrm{m}$. Right: $\omega=10 \mathrm{GHz}$ and $L=1 \mu \mathrm{m}$.

\begin{tabular}{|l|l|l|}
\hline Parameter & Physical meaning & Numerical value \\
\hline$L_{y} / L_{z}$ & extension of device in $y / z$-direction & $10^{-6} / 10^{-5} \mathrm{~m}$ \\
$U_{n} / U_{p}$ & band potentials in active region & $0.1 /-0.1 \mathrm{~V}$ \\
$B$ & spontaneous recombination parameter & $10^{-16} \mathrm{~m}^{3} / \mathrm{s}$ \\
$n_{t h}$ & threshold density & $10^{24} \mathrm{~m}^{-3}$ \\
$\alpha_{f} / \alpha_{b g}$ & mirror/optical background loss & $5000 / 4000 \mathrm{~m}^{-1}$ \\
$\epsilon_{s}^{A} / \epsilon_{s}^{G}$ & material permittivity & $1.08 \cdot 10^{-10} / 1.14 \cdot 10^{-10} \mathrm{As} / \mathrm{Vm}$ \\
$\mu_{n}^{A} / \mu_{n}^{G}$ & electron mobilities & $2300 / 8300 \mathrm{~cm}^{2} / \mathrm{Vs}$ \\
$\mu_{p}^{A} / \mu_{p}^{G}$ & hole mobilities & $145 / 400 \mathrm{~cm}^{2} / \mathrm{Vs}$ \\
$\mu_{o p t}^{A} / \mu_{o p t}^{G}$ & refractive index & $3.3 / 3.15$ \\
$n_{i}^{A} / n_{i}^{G}$ & intrinsic density & $2.1 \cdot 10^{9} / 2.1 \cdot 10^{12} \mathrm{~m}^{-3}$ \\
$g_{0}^{G}$ & differential gain in GaAs & $10^{-20} \mathrm{~m}^{2}$ \\
\hline
\end{tabular}

Table 2. Physical parameters for a laser diode of $\mathrm{Al}_{0.7} \mathrm{Ga}_{0.3} \mathrm{As}$ (superscript $A$ ) and GaAs (superscript $G$ ). Parameters without superscript are taken for both materials.

diode coupled to a high-pass filter (see Fig. 3). We have taken a capacitance of $10 \mathrm{pF}$, the resistances $R_{1}=1 \mathrm{M} \Omega, R_{2}=100 \Omega$, and $R_{3}=1 \mathrm{k} \Omega$, and a backward bias of $0.2 \mathrm{~V}$. The laser diode has the length of $1 \mu \mathrm{m}$ with an intrinsic region of $0.1 \mu \mathrm{m}$ length in the center of the device. The doping concentration is $-10^{24} \mathrm{~m}^{-3}$ in the $p$-doped region, $10^{24} \mathrm{~m}^{-3}$ in the $n$-doped region, and $10^{18} \mathrm{~m}^{-3}$ in the intrinsic region. The photo diode has a size of $L=6 \mu \mathrm{m}$, $L_{y}=10^{-5} \mathrm{~m}$ and $L_{z}=10^{-4} \mathrm{~m}$. For the quantum efficiency we assume $\eta=0.5$, for the surface reflectivity $r=0.3$ and $\alpha=5000 \mathrm{~m}^{-1}$ for the absorption. The remaining parameters are taken from Tables 1 and 2 .

In Fig. 3 the energy density in the laser diode during one half oscillation is shown. After having passed the threshold, the energy density increases tremendously in the active region. This is due to carrier confinement in the heterostructure, as in the lasing mode the carrier density is very high in the active region.

Finally, we operate the transmitter with a $1 \mathrm{GHz}$ digital signal of $2 \mathrm{~V}$. In Fig. 4 the light output signal and the received signal by the high-pass 

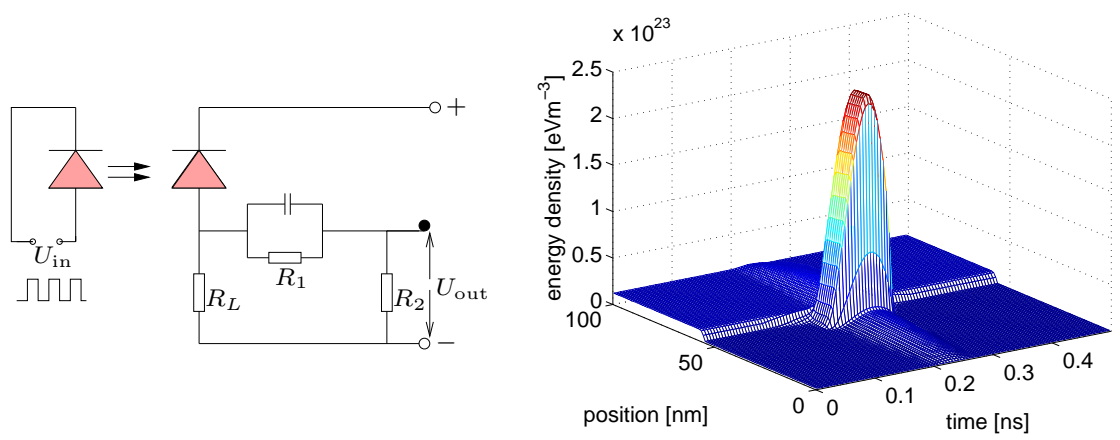

Fig. 3. Left: Laser and photo diode with a high-pass filter. Right: energy density in the laser diode for signal $v(t)=2 \sin \left(2 \pi t 10^{9}\right) \mathrm{V}$.
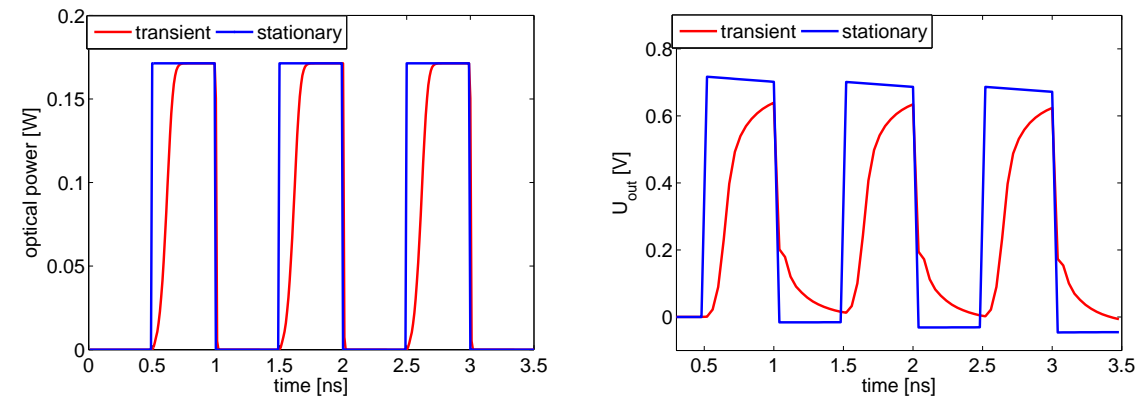

Fig. 4. Output of the laser diode and the high-pass filter for the stationary and transient energy-transport model with a digital input signal of $1 \mathrm{GHz}$.

filter is presented. Again we observe that the transient energy-transport model responds better to the capacitive effects in high-frequency circuits than the stationary model.

\section{Conclusion}

We have presented a coupled model consisting of the circuit equations from modified nodal analysis and the energy-transport model for semiconductor devices, resulting in a system of nonlinear partial differential-algebraic equations. This system allows for a direct simulation of thermal effects and can help to improve compact models of integrated circuits. The coupled model is tested on a Graetz circuit and a high-frequency transmitter with laser and photo diodes. The results show the impact of the thermal energy on the circuit. Compared to the constant-temperature drift-diffusion model, the output signal is smaller due to thermal effects. 
With decreasing size of the basic components in integrated circuits and special power devices, the thermal interaction between circuit elements will increase in importance in the near future. Therefore, we need to model not only the carrier temperature but also the device temperature and the interaction between the circuit elements. Thus, a heat equation for the temperature of the semiconductor lattice needs to be included in the presented model. This extension is currently under investigation. We expect that the resulting model will improve significantly the prediction of hot-electron effects and hot spots in integrated circuits.

\section{References}

1. A. M. Anile, V. Romano, and G. Russo. Extendend hydrodynamic model of carrier transport in semiconductors. SIAM J. Appl. Math. 61 (2000), 74-101.

2. U. Bandelow, H. Gajewski, and R. Hünlich. Fabry-Perot lasers: thermodynamicbased modeling. In: J. Piprek (ed.), Optoelectronic Devices. Advanced Simulation and Analysis. Springer, Berlin (2005), 63-85.

3. M. Bodestedt. Index Analysis of Coupled Systems in Circuit Simulatiom. Licentiate Thesis, Lund University, Sweden, 2004.

4. F. Brezzi, L. Marini, S. Micheletti, P. Pietra, R. Sacco, and S. Wang. Discretization of semiconductor device problems. In: W. Schilders and E. ter Maten (eds.), Handbook of Numerical Analysis. Numerical Methods in Electromagnetics. Elsevier, Amsterdam, Vol. 13 (2005), 317-441.

5. M. Brunk and A. Jüngel. Numerical coupling of electric circuit equations and energy-transport models for semiconductors. To appear in SIAM J. Sci. Comput., 2007.

6. M. Brunk and A. Jüngel. Simulation of thermal effects in optoelectronic devices using energy-transport equations. In preparation, 2007.

7. S. L. Chuang. Physics of Optoelectronic Devices. Wiley, New York, 1995.

8. P. Degond, A. Jüngel, and P. Pietra. Numerical discretization of energytransport models for semiconductors with non-parabolic band structure. SIAM J. Sci. Comp. 22 (2000), 986-1007.

9. M. Friedrich. Ein analytisches Modell zur Simulation von Silizium-Germanium Heterojunction-Bipolartransistoren in integrierten Schaltungen. PhD Thesis, Ruhr-Universität Bochum, Germany, 2002.

10. G. R. Jones, R. J. Jones, and W. French. Infrared HgCdTe Optical Detectors. In: J. Piprek (ed.), Optoelectronic Devices. Advanced Simulation and Analysis. Springer, Berlin (2005), 381-403.

11. R. Lamour. Index determination and calculation of consistent initial values for DAEs. Computers Math. Appl. 50 (2005), 1125-1140.

12. L. D. Marini and P. Pietra. New mixed finite element schemes for current continuity equations. COMPEL 9 (1990), 257-268.

13. C. Tischendorf. Modeling circuit systems coupled with distributed semiconductor equations. In: K. Antreich, R. Bulirsch, A. Gilg, and P. Rentrop (eds.), Modeling, Simulation, and Optimization of Integrated Circuits, Internat. Series Numer. Math. 146 (2003), 229-247.

14. A. Yamnahakki. Second-order boundary conditions for the drift-diffusion equations for semiconductors. Math. Models Meth. Appl. Sci. 5 (1995), 429-455. 